\title{
La "ontología histórica" de Michel Foucault. Apuntes de método para el análisis crítico socio-cultural
}

\author{
Pedro Cerruti \\ Doctor en Ciencias Sociales (Universidad de Buenos Aires) \\ Profesor en la Universidad de Buenos Aires \\ pedrocerruti@gmail.com
}

\begin{abstract}
Resumen El trabajo propone una reconstrucción de la perspectiva epistemológica y metodológica de Michel Foucault ordenada en función de lo que él mismo caracterizó al final de su vida como una "ontología histórica”, considerada aquí como el núcleo específico de un método original que se ha revelado como una herramienta hermenéutica fundamental para los diferentes modos de abordaje de las problemáticas socio-culturales contemporáneas. Para ello, se presenta una interpretación de algunos de los conceptos clave de las formulaciones de método explicitadas por el mismo Foucault, especialmente la "genealogía", el "acontecimiento" y los "modos de subjetivación", así como las "formas de experiencia", el "dispositivo" y la "problematización". Además, de los textos del propio Foucault, que constituyen la fuente primordial del trabajo, se recurre a las propuestas de lectura de algunos intérpretes que han realizado intervenciones particularmente indicativas respecto de dichos conceptos y problemas, como Gilles Deleuze, Giorgio Agamben y Mathieu Potte-Bonneville.
\end{abstract}

Palabras-clave: Michel Foucault, ontología histórica, análisis socio-cultural, genealogía, subjetivación.

\section{Introducción}

$\mathrm{D}$ ESDE HACE DÉCADAS, la obra de Foucault ha venido ejerciendo una notable influencia en el campo de las ciencias sociales, alcanzando una notable popularidad y generando una renovación del pensamiento crítico de una extensión solo proporcional con las dificultades que su pensamiento acarrea a la hora de intentar clasificarlo según las tradicionales fronteras disciplinares. Los métodos de Foucault, que involucran el reemplazo de las macro-categorías de análisis social, de los valores universales fundamentados ya sea en la moral o en la razón, de la mirada preocupada por los desarrollos teleológicos de las grandes transformaciones históricas, por una perspectiva fiel al análisis inmanente de los discursos, a la indagación en detalle de la producción de las formas culturalmente situadas de subjetivación, al estudio de las prácticas específicas entendidas en función de las redes de poder que en ellas se traman, y a una perspectiva del devenir histórico-social entendida en términos genealógicos y "acontecimientales", han incidido de modo significativo en el campo de los estudios socio-históricos (Roth, 1981; Dean, 1994; Potte-Bonneville, 2007), políticos (Barry, Osborne et al., 1996; Clifford, 2001), filosóficos (Balbier, Deleuze et al., 1990; 
O'Leary y Falzon, 2010) y antropológicos y culturales (Knauft, 1996; Neubauer, 1999; Bratich, Packer et al., 2003). Ello, que puede ser enunciado en términos generales, no vuelve superfluo reconocer que ha tenido inclusive un impacto en la rica y singular trayectoria de los estudios culturales latinoamericanos, arraigados en formas particulares del ensayismo y de la temprana recepción de la teoría crítica (Trigo, 2002; Szurmuk e Irwin, 2009).

Ya en lo que constituye el primer intento de sistematización de la obra de Foucault, publicada poco antes de su muerte por Dreyfus y Rabinow (2001), se refieren a su obra en los términos de un intento por desarrollar un método que, apuntalándose en los más importantes proyectos de comprensión de los seres humanos desarrollados durante el siglo XX -la fenomenología, la hermenéutica y el estructuralismo-, al mismo tiempo que ponen en cuestión los resultados de sus ambiciosos proyectos, busca de diferentes maneras a lo largo de su desarrollo ir más allá de ellos y construir una alternativa que, vista retrospectivamente, se ha revelado como de una enorme potencia heurística.

Sin embargo, la considerable heterogeneidad de las interpretaciones y aplicaciones de las propuestas metodológicas, las ideas y los conceptos foucaultianos, como bien afirman Moya y Andrew (1997), ha generado una bibliografía ya inabarcable que corre el riesgo de diluir la especificidad de un método original que consideramos constituye todavía una herramienta hermenéutica fundamental para los diferentes modos de abordaje de las problemáticas socio-culturales contemporáneas.

Por ello, más que considerar dicho "método" en términos del desarrollo de una doctrina o una metodología que pueda ser formalizada y luego aplicada en diferentes contextos, el punto de partida de este trabajo se encuentra en el reconocimiento de que el valor de la obra de Foucault reside fundamentalmente en su potencia como herramienta para problematizar nuestras prácticas intelectuales. ${ }^{1}$ En este sentido, vale la pena recordar el modo en que en ¿Qué es la Ilustración?, Foucault inscribe su propio trabajo en lo que denomina un "éthos filosófico" caracterizado por "una crítica permanente de nuestro ser histórico", es decir, un "tipo de interrogación filosófica que problematiza a la vez la relación con el presente, el modo de ser histórico y la constitución de sí mismo como sujeto autónomo" (Foucault, 1999, p. 345). Se trata entonces de una crítica permanentemente reactivada de lo que decimos, pensamos y hacemos que encuentra su principio en una "ontología histórica" de nosotros mismos. Es en esa actitud de reflexión históricocrítica, o de fidelidad a ese éthos, que liga en definitiva esta perspectiva de trabajo con la Aufklarung, en la que nos interesa detenernos aquí.

En esa "ontología histórica" que propone Foucault como fundamento epistemológico es donde se buscará situar la especificidad de una perspectiva de trabajo analítico que no ha perdido nada de su vigencia. En el presente trabajo se ha optado por presentar una lectura que atraviesa la obra de Foucault para situar, más allá de las diferentes etapas que escanden su trabajo en función de sus objetos y sus métodos particulares, ${ }^{2}$ la especificidad de dicha "ontología histórica". Para ello, se presentaran una serie de interpretaciones de algunos de los conceptos clave de las formulaciones de método explicitadas por el mismo Foucault, especialmente la "genealogía", el "acontecimiento" y los "modos de subjetivación", así como las "formas de experiencia", el "dispositivo" y la "problematización". Se recurrirá, además, a las propuestas de lectura de algunos de los intérpretes de Foucault que han realizado intervenciones particularmente indicativas respecto de dichos conceptos y problemas, como Gilles Deleuze, Giorgio Agamben y Mathieu Potte-Bonneville.

\footnotetext{
1. Este artículo es una reelaboración de contenidos que forman parte de la Tesis de Doctorado en Ciencias Sociales por la Universidad de Buenos Aires (UBA), específicamente de su fundamento epistémico- metodológico. La misma fue el producto de una investigación que se realizó en el marco de las becas de posgrado tipo I y II otorgadas por el Consejo Nacional de Investigaciones Científicas y Técnicas (CONICET, Argentina). El autor desea agradecer por ello a la UBA y al CONICET.

2. Los estudios sobre la obra de Foucault han establecido la posibilidad de reconocer que esta puede ser desagregada en tres diferentes períodos: un primer momento organizado en torno a la "arqueología" como método y a la pregunta en torno al saber, que en términos temporales se extiende desde 1961 a 1969 y, respecto de sus investigaciones, desde la Historia de la locura (1961) a La arqueología del saber (1969), pasando por El nacimiento de la clínica (1963) y Las palabras y las cosas (1966), entre otros; un segundo momento de 1971 a 1976 vinculado con sus investigaciones de tipo "genealógico", ordenadas alrededor de la pregunta por el poder, que se inicia con El orden del discurso (1970) y finaliza con el primer tomo de La historia de la sexualidad, La voluntad de saber (1976), e incluye al clásico Vigilar y castigar (1975); y un tercero dedicado al estudio de la ética, y orientada por el interrogante respecto de los modos de subjetivación, que se extiende hasta el final de su vida en 1984 y se ve plasmada principalmente en los dos últimos tomos de La historia de la sexualidad, El uso de los placeres (1984) y La inquietud de sí (1984) (Deleuze, 1987; Morey, 1990). Independientemente de las limitaciones inherentes a este tipo de periodizaciones, en el presente trabajo no nos detendremos en las especificidades de cada uno de esos momentos, sino que buscaremos precisar justamente la perspectiva particular que atraviesa toda su obra y que -como se ha indicado- en ¿Qué es la Ilustración?, uno de sus últimos textos, denomina con el sintagma "ontología histórica".
} 


\section{Las "formas de la experiencia" y el "dispositivo"}

En la primera clase de su curso en el Collège de France de 1983 titulado El gobierno de sí y de los otros, como era habitual al comienzo de cada nuevo ciclo, Foucault se detiene a realizar ciertas observaciones de índole metodológica. Allí, define a su trabajo como una "historia (crítica) del pensamiento" entendiendo por tal un análisis de "focos de experiencia" que articulan "formas de un saber posible", "matrices normativas de comportamiento de los individuos" y "modos de existencia virtuales para sujetos posibles" (Foucault, 2009, 19). La "locura", la "enfermedad", la "criminalidad" y la "sexualidad" se recortan justamente como "experiencias" a través de las cuales Foucault a lo largo de su obra profundizó el estudio de cada uno de esos ejes. Como tales, cada una de ellas no constituyen invariantes históricas que pueden ser representadas de diferentes maneras y en relación con las cuales se pueden adoptar diferentes actitudes sino que son el resultado de la correlación de "formas de saber", de "matrices de comportamientos" y de "modos de ser del sujeto".

En torno de cada uno de estos últimos ejes Foucault ordena lo que ha sido su estrategia de pensamiento. En primer lugar, se propone producir un abordaje de las "prácticas discursivas" que constituyen y ordenan matrices de conocimientos posibles en función del juego entre lo verdadero y lo falso, es decir, de las regulaciones que establecen las formas de la "veridicción". En segundo lugar, el estudio de las matrices normativas de comportamientos en términos de los ejercicios del poder y de las técnicas y los procedimientos de conducción de la conducta de los otros. Finalmente, el análisis de los modos a través de los cuales los individuos se constituyen en sujetos a través de una "pragmática de sí", o sea, de las tecnologías o técnicas de relación consigo mismo.

En el Prefacio a 'La historia de la sexualidad II' Foucault se refiere a su trabajo casi en los mismos términos como un "estudio de las formas de la experiencia en su historia", pero poniendo un mayor acento en el problema de la "subjetivación". Según sus palabras, "la tarea era sacar a la luz el dominio en el que la formación, el desarrollo, la transformación de las formas de experiencia pueden tener lugar; es decir, una historia del pensamiento. Por 'pensamiento' entiendo lo que instaura, en sus diferentes formas posibles, el juego de lo verdadero y de lo falso, y que, en consecuencia, constituye al ser humano como sujeto de conocimiento; lo que funda la aceptación o el rechazo de la regla y constituye al ser humano como sujeto social y jurídico; lo que instaura la relación consigo mismo y con los otros, y constituye al ser humano como sujeto ético" (Foucault, 1984, 334).

Es decir que la conformación de un "campo de experiencia" implica juegos de verdad que establecen una "objetividad"; relaciones de poder que determinan la constitución de una política y una "gubernamentalidad"; y modalidades de relación consigo mismo y con los otros, lo cual supone la elaboración de una ética y de una práctica de sí mismo.

El eje ordenador es la noción de "experiencia" entendida como las formas históricas de subjetivación, y que involucra planos cognitivos, jurídicos, sociales, éticos etc. Se entiende por qué, entonces, la noción de subjetivación domina el pensamiento de Foucault en los últimos años de su vida al punto de llegar a decir que "no es el poder, sino el sujeto, el tema general de mis investigaciones" y que el objetivo de su trabajo ha sido "crear una historia de los diferentes modos a través de los cuales, en nuestra cultura, los seres humanos se han convertido en sujetos" (Foucault, 2001, 241).

Ahora bien, de las nociones utilizadas por Foucault a lo largo de su obra probablemente sea la de dispositivo la que mejor permita operativizar dichos elementos. Es el mismo sentido en el que se ha manifestado el filósofo italiano Giorgio Agamben al proponer que la palabra dispositivo, que Foucault utiliza particularmente en sus estudios sobre la "gubernamentalidad" a fines de los años setenta, constituye un "término técnico" decisivo en su estrategia de pensamiento pues constituye un concepto operativo de carácter general que ocupa el lugar de las categorías con pretensión de universalidad cuya recusación es al mismo tiempo un prerrequisito metodológico y un efecto de esta perspectiva (Agamben, 2006). ${ }^{3}$

Ahora bien, el mismo Foucault había sido sometido ya a la pregunta de qué es un dispositivo en una entrevista realizada en 1977 y publicada bajo el título El juego de Michel Foucault (Foucault, 1985). Allí lo define destacando tres aspectos. En primer lugar, un dispositivo consiste en "un conjunto resueltamente heterogéneo, que implica discursos, instituciones, disposiciones arquitectónicas, decisiones reglamentarias, leyes, medidas administrativas, enunciados administrativos; proposiciones filosóficas, morales, filantrópicas; en síntesis, tanto lo dicho cuanto lo no dicho" (Foucault, 1985, 184). En segundo lugar, lo que define a los dispositivos es "la naturaleza del vínculo que puede existir entre esos elementos heterogéneos", es

3. Agamben retoma la perspectiva abierta por Gilles Deleuze en su intervención en el Encuentro Internacional organizado en el año 1988 por la Association pour le Centre Michel Foucault, titulada "¿Qué es un dispositivo?" y publicada en el volumen colectivo Michel Foucault, filósofo (Deleuze, 1990) -sobre la que nos detendremos más adelante. Que Agamben elija el mismo título para su conferencia es suficientemente indicativo de esta deuda. 
decir, que "entre estos elementos -discursivos y no discursivos- existe algo así como un juego, cambios de posición, modificaciones de funciones, que pueden, también ellos, ser muy diferentes" (Foucault, 1985, 184). El tercer elemento es el "imperativo estratégico" que constituye su matriz, con lo cual un dispositivo es "una especie de formación que, en un momento histórico dado, ha tenido como función principal responder una urgencia" (Foucault, 1985, 185).

En síntesis, un dispositivo es una red que puede establecerse entre elementos discursivos y no discursivos, en otras palabras, entre discursos y prácticas, o entre determinados saberes y ciertas relaciones de fuerza, en cuyo surgimiento está puesta en juego la necesidad de responder a un problema.

Decir que el dispositivo es de "naturaleza esencialmente estratégica" o que "tiene una función estratégica dominante" quiere decir que su definición y análisis es inseparable de su "génesis". Al respecto, Foucault señala la posibilidad de reconocer distintos "momentos" y "procesos" en dicha génesis. A un primer tiempo de "predominio de un objetivo estratégico", le sigue un doble proceso de "sobredeterminación causal" y de "ocupación estratégica". Por "sobredeterminación causal" se entiende un desarrollo en función del cual cada efecto producido por el dispositivo, independientemente de que haya sido deseado o no, o de que sea positivo o negativo, entra en relaciones de resonancia o contradicción con los otros efectos producidos, de tal manera que lleva a desajustes y recuperaciones, desequilibrios y nuevas compensaciones entre los diferentes elementos heterogéneos que no cesan de surgir. Por su parte, la "ocupación estratégica" es un proceso constante de reutilización, con fines diversos, de los efectos del dispositivo, incluso aquellos negativos, imprevisibles o involuntarios, dándoles una nueva positividad en el seno de estrategias novedosas.

En conclusión, es posible utilizar el término dispositivo para nombrar a los "conjuntos prácticos" con los que en ¿Qué es la ilustración? Foucault designaba de modo genérico el objeto específico de los estudios genealógicos, y concebirlos, por lo tanto, como articulando una faz "tecnológica", es decir, formas de racionalidad que organizan las maneras de hacer; y una faz "estratégica", definida por el "juego de libertades" por el cual los sujetos actúan reaccionando frente a los otros y modificando las reglas de dicha organización.

\section{La "genealogía" y la "acontecimientación"}

La genealogía puede entenderse, entonces, como una modalidad de ejercicio de la investigación histórico-crítica que procede por medio del análisis de los dispositivos. Esto quiere decir que no lo hace mediante la búsqueda de las estructuras formales de carácter universal que organizarían el conocimiento y la acción; sino que lo hace mediante el estudio de los acontecimientos históricos para reconocer allí el modo en que se ha construido un campo de experiencia y la forma en que nos hemos constituido como sujetos de lo que decimos, pensamos y hacemos.

Se trata, por ello, de un procedimiento de "acontecimientación", según el neologismo inventado por Foucault. En la intervención publicada con el nombre de Debate con los historiadores, esta es definida en primera instancia como una "ruptura de evidencia": "allí donde se estaría ciertamente tentado a referirse a una constante histórica o a un rasgo antropológico inmediato, o aun a una evidencia que se impone del mismo modo a todos, se trata de hacer surgir una 'singularidad"' (Foucault, 1985, 220). La tarea consiste en mostrar que aquello que se nos presenta como necesario, universal o evidente no es tal, es decir, en una "ruptura de las evidencias sobre las que se apoya nuestro saber, nuestras aquiescencias, nuestras prácticas" (Foucault, 1985, 220); y hacerlo por la vía de reconstruir los procesos (las relaciones de fuerza, los juegos de verdad, las estrategias, las resistencias etc.) que un momento determinado han hecho posible aquello que luego va a funcionar como evidencia, universalidad o necesidad. Ello supone un trabajo de "desmultiplicación causal", o sea, un análisis de los múltiples procesos que constituyen el acontecimiento, que se asemeja a construir a su alrededor "un 'poliedro' de inteligibilidad cuyo número de caras no ha sido definido previamente ni puede jamás ser considerado como acabado de pleno derecho" (Foucault, 1985, 221); y hace necesario contemplar el "polimorfismo" tanto de los elementos que se ponen en relación, como de las relaciones descriptas y de los dominios de referencia.

Es por esa razón que ya en su clásico Nietzsche, la genealogía, la historia, Foucault comenzaba destacando como la "tarea indispensable" de la genealogía "percibir la singularidad de los sucesos, fuera de toda finalidad monótona; encontrarlos allí donde menos se lo espera y en aquello que pasa desapercibido por carecer de historia [...]; captar su retorno, pero en absoluto trazar la curva lenta de una evolución, sino reencontrar las diferentes escenas en las que han jugado diferentes papeles; definir incluso el punto de su ausencia, el momento en que no han tenido lugar" (Foucault, 1992, 7). 
Frente a la búsqueda del "despliegue metahistórico de las significaciones", de los "fundamentos originarios" y las "causas finales", dice Foucault, "la historia 'efectiva' hace surgir el suceso en lo que puede tener de único, de cortante" (Foucault, 1992, 21).

\section{El "dispositivo" según Gilles Deleuze}

Puede decirse, entonces, que la noción de dispositivo, anclada en los estudios específicamente genealógicos sobre la prisión (Foucault, 1999), la sexualidad (Foucault, 2002) y la gubernamentalidad (Foucault, 2006), incorpora la perspectiva arqueológica que guió las investigaciones sobre la locura (Foucault, 1976), la clínica (Foucault, 2001) y las ciencias humanas (Foucault, 1989). El mismo Foucault señala que el concepto de "episteme" debe ser introducido en este nuevo punto de vista a partir del cual puede ser redefinida como un "dispositivo específicamente discursivo". ${ }^{4}$ El dispositivo posee, pues, una carácter más general ya que está, en sus palabras, "inscripto en un juego de poder, pero también siempre ligado a uno o unos bornes de saber que nacen allí pero que igualmente lo condicionan. Eso es un dispositivo: unas estrategias de relaciones de fuerzas soportando unos tipos de saber, y soportadas por ellos" (Foucault, 1985).

A su vez, la noción de dispositivo puede abrirse para incorporar los estudios posteriores ordenados en torno a la noción de subjetividad. Justamente Gilles Deleuze, en la intervención mencionada, ha ofrecido, desde una mirada retrospectiva de la obra de Foucault, una síntesis de los elementos que convergen en una definición posible de los dispositivos, al mismo tiempo que ha situado la importancia que la noción de subjetivación tiene para cualquier pensamiento que se proponga el análisis de los mismos.

"Saber", "poder" y "subjetividad", las tres instancias que escanden la obra de Foucault, son consideradas por Deleuze como "vectores" o "tensores" en un "conjunto multilineal" que, lejos de constituir un sistema, componen un "ovillo" o una "madeja". La genealogía consiste, parafraseando a Deleuze, en "desenmarañar" las líneas que componen esa madeja informe, inacabada y dinámica que es un dispositivo.

Los dispositivos, en la lectura de Deleuze, están compuestos por líneas de visibilidad y enunciación en función de las cuales son pensables como "máquinas para hacer ver y hacer hablar" (Deleuze, 1990, 155). Pero es necesario destacar que la "visibilidad" no se refiere al hecho de iluminar objetos dados o preexistentes, sino que esas líneas forman figuras que son variables e inseparables de las mismas: "cada dispositivo tiene su régimen de luz, la manera en que esta cae, se esfuma, se difunde, al distribuir lo visible y lo invisible, al hacer nacer o desaparecer el objeto que no existe sin ella" (Deleuze, 1990). Al mismo tiempo, las enunciaciones, provenientes ya sean del campo científico, literario, jurídico, político o social, remiten a un régimen de lo enunciable, es decir, a "líneas de enunciación en las que se distribuyen las posiciones diferenciales de sus elementos", siendo al mismo tiempo ellas mismas, en tanto que enunciaciones, "curvas que distribuyen variables [...] con sus derivaciones, sus transformaciones, sus mutaciones" (Deleuze, 1990, 156).

Por otra parte, los dispositivos implican "líneas de fuerza" que componen la "dimensión del poder". Dice Deleuze: "parecería que estas fueran de un punto singular a otro situado en las líneas precedentes; de alguna manera 'rectifican' las curvas anteriores, trazan tangentes, envuelven los trayectos de una línea con otra, operan idas y venidas, desde el ver al decir e inversamente, actuando como flechas que no cesan de penetrar las cosas y las palabras, que no cesan de librar una batalla" (Deleuze, 1990, 156). El poder atraviesa todos los elementos del dispositivo siendo él mismo "invisible e indecible", siempre entremezclado con las otras líneas al punto de ser indistinguible.

Junto con ellas, los dispositivos implican "líneas de subjetivación", es decir, regímenes por medio de los cuales los seres humanos se convierten en sujetos. Pero la noción de subjetivación ocupa allí un lugar particular que Deleuze expresa de la siguiente forma: "este descubrimiento [de los modos de subjetivación] nace de una crisis producida en el pensamiento de Foucault, como si este hubiera tenido que modificar el mapa de los dispositivos, encontrarles una nueva orientación posible, para no dejarlos que se cerraran simplemente en líneas de fuerza infranqueables, que impusieran contornos definitivos" (Deleuze, 1990, 156).

Lo que le interesa destacar es que, así como los sujetos son un producto de los dispositivos, las líneas de subjetivación escapan a las determinaciones de los mismos. La producción de subjetividad es, dice Deleuze, "un proceso de individuación que tiene que ver con grupos o personas y que se sustrae a las relaciones de fuerzas establecidas como saberes constituidos" (Deleuze, 1990, 157). Con toda razón, agrega que "se preguntará uno si las líneas de subjetivación no son el borde extremo de un dispositivo y si ellas no esbozan el paso de un dispositivo a otro" (Deleuze, 1990, 157). En ese sentido los procesos de subjetivación, inmanentes al dispositivo, constituyen al mismo tiempo sus líneas de fuga o de fractura. Si la noción 
de dispositivo ocupa el lugar de los "universales" que Foucault recusa, el énfasis puesto en el problema de la subjetivación es fundamental para que la noción misma no se erija como un nuevo trascendental; para no confundirla con una estructura formal que, inmutable más allá de los fenómenos, nos daría el criterio de su inteligibilidad y bajo la cual el sujeto está destinado a desaparecer; ni para ver en él, en palabras de Foucault, la "astucia estratégica de algún sujeto meta o transhistórico que lo habría percibido o querido" (Foucault, 1985, 185). Ello tampoco implica, desde luego, hacer de la subjetividad el lugar para un nuevo mito emancipatorio sino simplemente considerar el hecho de que "las producciones de subjetividad se escapan de los poderes y de los saberes de un dispositivo para colocarse en los poderes y saberes de otro, en otras formas por nacer" (Deleuze, 1990, 157).

\section{La "problematización"}

Como se ha adelantado, las elecciones metodológicas de Foucault suponen un escepticismo respecto de todas las categorías que pretenden una validez universal. Categorías como "locura", "delincuencia" o "sexualidad" son categorías cuyo contenido varía históricamente y el trabajo de análisis consiste en interrogar las condiciones, que en función de determinadas reglas de veridicción, hacen posible, o incluso necesario, reconocer en ellas a determinados sujetos. Lo cual no quiere decir que deban ser rechazadas como meras quimeras o como nociones que no se refieran a nada. Se trata en todo caso, en palabras de Foucault, de "elidir tanto como sea posible para interrogarlos en su constitución histórica, los universales antropológicos (entendiendo por tales los de un humanismo que hiciera valer los derechos, los privilegios y la naturaleza de un ser humano como verdad inmediata e intemporal del sujeto)" (Foucault, 1999, 366).

Al mismo tiempo, implican rechazar el recurso a un "sujeto constituyente" que podría dar cuenta de la conformación de un determinado campo de experiencia. Nuevamente, no se trata de descartar al sujeto como si este no existiera para acceder a un registro de objetividad pura. Lo que está en juego es la posibilidad de acceder a un estudio de las prácticas mediante las cuales el sujeto se constituye en la inmanencia de los dispositivos.

Es necesario, en consecuencia, conservar el carácter aporético de esta perspectiva, que puede enunciarse en los siguientes términos: "estos juegos [de verdad] no se imponen desde el exterior al sujeto, de acuerdo con una causalidad necesaria o con determinaciones estructurales; abren un campo de experien- cia en el que el sujeto y el objeto no se constituyen uno y otro sino bajo ciertas condiciones simultáneas, pero en las que, a su vez, no dejan de modificarse el uno con relación al otro, y por tanto de modificar ese mismo campo de experiencia" (Foucault, 1999).

Es decir, una conformación correlativa del objeto y el sujeto cuya clave de inteligibilidad reside justamente en las "prácticas", entendidas como los modos en los que se ha constituido tanto aquello que se coloca como el objeto "real" para el pensamiento como los sujetos capaces de conocerlo, analizarlo e intervenir sobre él. En definitiva, se trata de "hacer aparecer los procesos peculiares de una experiencia en la que el sujeto y el objeto 'se forman y se transforman', uno por relación y en función del otro" (Foucault, 1999).

Resulta, entonces, comprensible que Foucault pueda hablar de su trabajo en términos de una "genealogía de las problematizaciones" (Foucault, 2003, 54), es decir, no una historia de las soluciones que se han ensayado como respuesta para resolver ciertos problemas, sino de los modos en que un comportamiento, un campo de acción, una experiencia etc., que a partir de determinado momento resulta incierta y motivo de dificultades, ha sido "problematizado" por el trabajo del pensamiento. Y esa manera de problematización es el suelo sobre el cual germinan las diferentes soluciones. Por supuesto que hay procesos sociales, económicos, políticos etc., que incitan este proceso, pero no determinan el modo efectivo de problematización ya que cuando el pensamiento interviene "no adopta una forma única que será el resultado directo o la expresión necesaria de estas dificultades; es una respuesta original o específica a menudo multiforme, a veces incluso contradictoria en sus diferentes aspectos, a esas dificultades que son definidas por él mediante una situación o un contexto y que valen como una cuestión posible" (Foucault, 1999, 360).

En otras palabras, "se pueden dar varias respuestas a un mismo conjunto de dificultades [...] lo que hay que comprender es lo que las hace simultáneamente posibles" (Foucault, 1999, 360). Eso es su forma general de problematización, la cual responde a determinadas dificultades o urgencias no elaborando respuestas a las mismas sino definiendo como tal el problema al que las soluciones buscarán responder y, por ello, haciéndolas posibles. Por lo tanto, su lógica no es la del descubrimiento, la revelación o la adquisición de nuevas verdades sino la de la emergencia, es decir, la de las formas singulares de aparición de los juegos de verdad que a través de una objetivación y una subjetivación correlativas articulan cierto tipo de objetos a determinadas modalidades del sujeto. Se trata de entender de qué manera "dicha emergencia ha constituido, para un tiempo, para un área y para individuos dados, el a priori histórico de una expe- 
riencia posible" (Foucault, 1999, 364). Las problematizaciones no son, pues, ni una constante antropológica ni una variación cronológica.

El proyecto de Foucault puede ser concebido, entonces, como un estudio genealógico que se despliega en tres ámbitos: el eje del saber, en función del cual se establecen nuestras relaciones con las cosas; el eje del poder, es decir, el de nuestras relaciones con los otros; y el eje de la ética, o el de las relaciones consigo mismo. Por lo tanto, presupone una ontología histórica de nosotros mismos en tanto que sujetos de saber, sujetos en un campo de poder y sujetos de una moral.

En síntesis, el trabajo genealógico exige sustituir la referencia a categorías universales -ya sean la "locura", el "crimen", la "sexualidad", como también el "Estado", la "Ley", el "Poder" o la "Justicia"- por el análisis de experiencias que constituyen formas históricas singulares; y reemplazar una teoría del conocimiento, del poder o del sujeto por el análisis de prácticas - discursivas y no discursivas- históricas determinadas. A su vez, requiere prescindir de la pretensión de adecuar las prácticas a sistemas de valores que permitan dar cuenta de ellas e inscribir esos sistemas en el juego de prácticas inteligibles pero contingentes (Foucault, 2009, 22).

De lo antedicho se desprende, como última indicación, que si se entiende por "experiencia" la correlación entre dominios de saber, tipos de normatividad y modalidades de subjetivación; y si llamamos "pensamiento" a la "acción" que instaura las condiciones de esa experiencia; en palabras de Foucault, "no hay que buscar el pensamiento en las formulaciones teóricas, como las de la filosofía o de la ciencia; este puede y debe ser analizado en todas las maneras de decir, de hacer, de conducirse, en las que el individuo se manifiesta y obra como sujeto de conocimiento, como sujeto ético o jurídico, como sujeto consciente de sí y de los otros" (Foucault, 1984, 334-335).

La genealogía, al reconducir las "problematizaciones" que se nos presentan como las matrices necesarias de nuestra experiencia a sus formas de emergencia, es una práctica donde el análisis histórico y la crítica política se encuentran. Y lo hacen en una reflexión sobre los "límites", ya que el reconocimiento de lo que hay de singular, contingente y arbitrario en lo que se nos da como universal, necesario y obligatorio, dice Foucault, transforma a la crítica en una "forma de franqueamiento posible". En ese sentido, la genealogía "no deducirá de la forma de lo que somos lo que nos es imposible hacer o conocer, sino que extraerá de la contingencia que nos ha hecho ser lo que somos la posibilidad de ya no ser, hacer o pensar lo que somos, hacemos o pensamos" (Foucault, 1999, 348).

Pero es necesario aclarar que cuestionar a través de la actividad crítica los "mitos" que hacen de la his- toria "naturaleza" no conduce a una celebración sin más de aquello que se postula como cambio o novedad histórica. Al contrario, no se trata de determinar qué es "bueno" y qué es "malo" sino que la perspectiva que propone Foucault es la de que "todo es peligroso" y que, por lo tanto, "la elección ético-política que debemos hacer diariamente es determinar cuál es el principal peligro" (Foucault, 2003, 54). En otros términos, no existe un conocimiento completo de nuestros límites históricos, y por lo tanto tampoco hay un acceso definitivo a su franqueamiento; por lo cual la experiencia teórico-práctica que hacemos de ellos es siempre limitada, determinada y, por consiguiente, está permanentemente destinada a comenzar de nuevo.

\section{La "ontología histórica": subjetivación y libertad}

El filósofo francés Mathieu Potte-Bonneville, en su lectura de la obra de Foucault, ha puesto el acento justamente en las tensiones que la atraviesan y en la fidelidad a una modalidad de trabajo que no las elimine sino que las sostenga en lo que ellas tienen de fecundas.

Estas son aquellas que se establecen, por un lado, en el modo en que Foucault piensa las normas históricas en términos de la descripción inmanente de un orden de positividades y en contra de los diferentes tipos de mitos esencialistas y continuidades dialécticas que lo niegan; pero al mismo tiempo centrando sus estudios en aquellos objetos que permiten pensar las formas de la experiencia como preguntas sin respuestas. Y, por otro lado, en el lugar que le confiere a la instancia subjetiva no dándole lugar a un sujeto trascendente, posible de considerarse universal y de exceptuarse de la sociedad y de la historia; ni como mera particularización de las normas sociales o actualización de las estructuras atemporales. Es decir, pensar a los sujetos ni como fundamento ni como fin sino como emergencia de una singularidad en la historia.

En sus palabras, se trata de un método concentrado en "el esfuerzo por pensar, en términos de una descripción positiva, los puntos problemáticos donde discursos y prácticas escapan a ellos mismos; [y en] el esfuerzo por concebir, en el interior de dispositivos que ninguna conciencia inaugura, la emergencia de sujetos" (Potte-Bonneville, 2007, 20). De ahí la importancia otorgada por Foucault a los "puntos de problematización" a través del estudio de objetos -como la "locura", el "crimen" y la "sexualidad"- que constituyen los "desajustes" que son el centro y el efecto de la producción de las normas históricas, al mismo 
tiempo que se revelan como su límite; "zonas oscuras" que lejos de establecer un exterior de la historia o un caos ininteligible componen un espacio regulado en torno a las grietas donde esas normas se confrontan con su insuficiencia; ámbitos donde se ponen en suspenso las pretensiones de dominio y de autocomprensión que impulsan el "progreso" de la razón y donde la identidad del hombre se fractura; territorios donde al enfrentarse con aquello que se les resiste, pero que constituye su punto de apoyo, las normas reverberan hasta hacerse accesibles al análisis genealógico. En definitiva, se trata de "una historización de lo verdadero apoyada en una serie de preguntas" (Potte-Bonneville, 2007, 28).

En síntesis, los "puntos de problematización" son las experiencias, en tanto que ellas se constituyen como objetos en el marco de los dispositivos, es decir, al interior de una matriz de normatividad, pero al mismo tiempo donde esos juegos de verdad se confrontan con aquello que les es irreductible y donde, por lo tanto, se exponen y se difractan. Esos puntos de problematización son las fisuras por donde se producen las emergencias, los resquicios por donde surgen las singularidades, los huecos a través de los cuales se despliegan los acontecimientos.

Así, propone Potte-Bonneville, "si se trata, en Foucault, de pensar lo positivo en el doble sentido de lo efectivo y de lo fecundo, también se trata de hacer surgir allí, como problema central, las discontinuidades, fallas, en pocas palabras, los elementos de negatividad que, al volver al saber y al discurso inadecuados para sus propios principios dan por resultado algunas hiancias peligrosas" (Potte-Bonneville, 2007, 17).

$\mathrm{Y}$, al mismo tiempo, de "pensar al sujeto no como una instancia plena que daría sentido a la historia, sino como la asunción de los espacios que esta abre, como la estilización de los problemas que plantea, como la confrontación con lo impensable que suscita y conlleva" (Potte-Bonneville, 2007, 20). Esa era la conclusión de Foucault a propósito de la genealogía y la historia: "nadie es pues responsable de una emergencia, nadie puede vanagloriarse de ella; esta se produce en el intersticio" (Foucault, 1992, 17).

En definitiva, el concepto de "problematización" designa el pivote de los procesos de subjetivación y al mismo tiempo caracteriza reflexivamente lo que se pone en juego en el procedimiento genealógico. En otras palabras, allí se trata de "mostrar, a partir del archivo, que la subjetividad procede de las crisis y permanece vinculada con ellas; pero pensar, para ello, las normas y sus crisis desde una posición de subjetividad resquebrajada en sí misma, recorrida por los sacudimientos del presente: esta puede ser la extraña torsión del círculo hermenéutico que nos queda por aprender de Foucault" (Potte-Bonneville, 2007, 20).
En efecto, esa vuelta sobre sí de la problematización es necesaria para pensar el cuestionamiento del sujeto hacia sus propias prácticas sin por ello postular una subjetividad exceptuada del contexto histórico al que pertenece e interroga.

La actividad crítico-histórica integra, por tanto, la genealogía de las problematizaciones en tanto que historia de las maneras en que nos hemos interrogado y constituido como sujetos; y forma parte, por lo tanto, de la actividad del "pensamiento". Justamente de allí se desprende su función teórico-política. Recordemos las palabras de Foucault en Polémica, política, problematizaciones: "Lo que distingue al pensamiento es que es algo completamente diferente del conjunto de las representaciones que sustentan un comportamiento; es otra cosa que el dominio de las actitudes que lo pueden determinar. El pensamiento no es lo que habita una conducta y le da un sentido; es, más bien, lo que permite tomar distancia con relación a esta manera de hacer o de reaccionar, dársela como objeto de pensamiento e interrogarla sobre su sentido, sus condiciones y sus fines. El pensamiento es la libertad con respecto a lo que se hace, el movimiento mediante el cual nos desprendemos de ello, lo constituimos como objeto y lo reflejamos como problema" (Foucault, 1999, 359).

Resulta comprensible que Foucault no se proponga hacer una historia de las soluciones ni proponer posibles alternativas. El pensamiento no es un desarrollo necesario, no responde definitivamente a las preguntas que formula, ni funda nuevas certezas. Es una "actividad problematizante" de aquellas experiencias que se constituyen como preguntas y en torno a las cuales se elaboran categorías y se proponen soluciones que no son nunca definitivas. "El trabajo de una historia del pensamiento sería reencontrar en la raíz de estas diversas soluciones la forma general de problematización que las ha tornado posibles" (Foucault, 1999, 360), es decir, constituye una modalidad específica de elaborar los problemas que ciertas experiencias nos plantean.

Esa brecha insalvable entre las preguntas y las respuestas, esa zona de libertad donde se abre la posibilidad de desasirse de lo que se nos presenta como universal, necesario, obligatorio, pero que es al mismo tiempo el lugar donde residen los peligros, ese intersticio es el espacio de la constitución del sujeto de la problematización.

En definitiva, afirma Foucault: "caracterizaría, por tanto, el éthos filosófico propio de la ontología crítica de nosotros mismos como una prueba histórico-práctica de los límites que podemos franquear, y por consiguiente, como el trabajo de nosotros mismos sobre nosotros mismos en nuestra condición de seres libres" (Foucault, 1999, 249). 


\section{A modo de cierre}

En el presente trabajo se ha propuesto una reconstrucción de la perspectiva epistemológica y metodológica de Foucault ordenada en función de lo que él mismo caracterizó al final de su vida, y mirando retrospectivamente su trabajo, como una "ontología histórica". Desde este punto de vista se ha ubicado la noción de "experiencia" como el objeto de estudio de un trabajo de análisis histórico-crítico. Dicha noción permite abarcar los diferentes objetos particulares sobre los cuales se centraron las investigaciones de Foucault y articular así en un mismo concepto a los interrogantes que sus sucesivos trabajos fueron recortando: la pregunta por el saber y las prácticas discursivas que lo hacen posible; por el poder y las matrices normativas que organizan sus formas de ejercicio; $y$ por el sujeto y las técnicas de conducción de la conducta en función de las cuales este se produce. A su vez, se ha destacado que la noción de "experiencia" sitúa la última de estas preguntas como aquella que permite considerar a las otras en términos de diferentes formas de subjetivación vinculadas ya sea con el conocimiento y la verdad, el poder y las normas o el gobierno de la conducta y la ética.

A su vez, se ha propuesto la noción de "dispositivo" como un concepto operativo a los fines de la indagación crítica de la conformación histórica de los "focos de experiencia", en tanto permite abordar los conjuntos de prácticas, tanto discursivas como extradiscursivas, que emergen en un determinado momento histórico cumpliendo una función estratégica y cuya organización involucra relaciones de poder, formas de saber y modalidades de subjetivación específicas. Por su parte, el método de estudio de los dispositivos en su historia -es decir, en su génesis, sus ocupaciones estratégicas y sus sobredeterminaciones causales-, es la "genealogía", entendida ella como un procedimiento de "acontecimientación", esto es, de indagación de los momentos históricos como "acontecimientos" definidos en tanto tales por el modo en que producen la emergencia de singularidades en las prácticas que conforman los campos de experiencia.

Las "experiencias" constituyen, pues, formaciones históricas singulares, y su indagación, por medio de la genealogía, requiere sustituir la referencia a categorías universales por el análisis de prácticas específicas, esto es, por las diferentes maneras de decir, de hacer y de conducirse en función de las cuales los seres humanos se producen como sujetos. En ese sentido, las "experiencias" son el producto de diferentes formas de "problematización", es decir, de las formas singulares y contingentes en las que en un determinado momento histórico, y cumpliendo una función estratégica, la emergencia de determinados juegos de verdad y de relaciones de poder constituyen a las experiencias como tales y generan al mismo tiempo a los objetos y sujetos de las mismas.

La "ontología histórica" así entendida implica, además, una dimensión ético-política ya que al considerar las formas de subjetivación como emergencias históricas singulares y contingentes, y al desmontar su necesariedad, abre una brecha de libertad que habilita un franqueamiento posible, aunque nunca total ni definitivo, de los límites que conforman nuestras formas de experiencia.

Desde esta perspectiva es posible comprender, entonces, el análisis crítico social y cultural como una forma particular de problematización de nuestras experiencias y que, por lo tanto, incorpora una doble dimensión cognoscitiva, vinculada con una perspectiva ontológica que considera a sus objetos de estudio y a los sujetos que los indagan en su correlativa constitución histórica; y una dimensión ética vinculada con el reconocimiento de nuestras prácticas intelectuales como una interrogación de nuestros propios límites históricos y, por lo tanto, como un trabajo de pensamiento indefectiblemente comprometido con el horizonte de su tiempo. 


\section{Referencias}

AGAMBEN, G. Che cos' è un dispositivo? Roma: Nottetempo, 2006.

BALBIER, E., DELEUZE, G., FRANK, E., et al. Michel Foucault, filósofo. Barcelona: Gedisa,1990.

BARRY, A., OSBORNE, T., ROSE, N. Foucault and political reason. Liberalism, neo-liberalism and rationalities of government. Chicago: The University of Chicago Press, 1996.

BRATICH, J., PACKER, J., MCCARTHY, C. Foucault, cultural studies, and governmentality. New York: State University of New York Press, 2003.

CLIFFORD, M. Political genealogy after Foucault: savage identities. New York: Routledge, 2001.

DEAN, M.. Critical and effective histories: Foucault's methods and historical sociology. New York: Routledge,1994.

DELEUZE, G. Foucault. Barcelona: Paidós, 1987.

DELEUZE, G. ¿Qué es un dispositivo? In: Michel Foucault, filósofo. Barcelona: Gedisa,1990.

FOUCAULT, M. Historia de la locura en la época clásica, v. I y II. México: Fondo de Cultura Económica, 1976.

FOUCAUlT, M. Preface to The History of Sexuality. In: RABINOW, Paul. The Foucault Reader. New York: Pantheon, 1984.

FOUCAULT, M. Debate con los historiadores. In: El discurso del poder. Buenos Aires: Folios, 1985.

FOUCAULT, M. El juego de Michel Foucault. In: El discurso del poder. Buenos Aires: Folios, 1985.

FOUCAULT, M. Las palabras y las cosas: una arquelogía de las ciencias humanas. México: Siglo XXI, 1989.

FOUCAULT, M. Nietzsche, la genealogía y la historia. In: Microfísica del poder. Madrid: La Piqueta, 1992.

FOUCAULT, M. Foucault. In: Estética, ética y hermenéutica. Obras esenciales, Vol. III. Barcelona: Paidós, 1999.

FOUCAULT, M. Polémica, política, problematizaciones. In: Estética, ética y hermenéutica. Obras esenciales, Vol. III. Barcelona: Paidós, 1999.
FOUCAULT, M. ¿Qué es la Ilustración? In: Estética, ética y hermenéutica. Obras esenciales, Vol. III. Barcelona: Paidós, 1999. FOUCAULT, M. Vigilar y castigar. México: Siglo XXI, 1999.

FOUCAULT, M. El nacimiento de la clínica. Una arqueología de la mirada médica. México: Siglo XXI, 2001.

FOUCAULT, M. El sujeto y el poder. In: DREYFUS, Hubert L., RABINOW, Paul. Michel Foucault: más allá del estructuralismo y la hermenéutica. Buenos Aires: Nueva Visión, 2001.

FOUCAULT, M. Historia de la sexualidad 1: La voluntad de saber. Buenos Aires: Siglo XXI, 2002.

FOUCAULT, M. Genealogía de la ética. In: El yo minimalista y otras conversaciones. Buenos Aires: La Marca, 2003. FOUCAULT, M. Seguridad, territorio y población. Buenos Aires: FCE, 2006.

FOUCAULT, M. El gobierno de sí y de los otros. Buenos Aires: FCE, 2009.

KNAUFT, B. Genealogies for the present in cultural anthropo$\log \gamma$. New York: Routledge, 1996.

MOREY, M. Introducción: la cuestión del método. In: FOUCAULT, Michel. Tecnologías del yo y otros textos afines. Barcelona: Paidós, 1990.

NEUBAUER, J. Cultural history after Foucault. New York: Aldine de Gruyter, 1999.

O'LEARY, T., FALZON, C. Foucault and philosophy. Oxford: Blackwell, 2010.

POTTE-BONNEVILLE, M. Michel Foucault, la inquietud de la historia. Buenos Aires: Manantial, 2007.

ROTH, M. S. Foucault's 'History of the Present'. History and Theory, n. 20, v. 1, 1981.

SZURMUK, M., IRWIN, R. Presentación. In: Diccionario de estudios culturales latinoamericanos. Mexico: Siglo XXI, 2009. TRIGO, B. Foucault and Latin America: appropriations and deployments of discoursive analysis. New York: Routledge, 2002. 


\title{
The "historical ontology" of Michel Foucault: methodological notes for critical socio-cultural analysis
}

\begin{abstract}
This paper proposes a reconstruction of Michel Foucault's epistemological and methodological perspective, organized according to what he characterized, at the end of his life, as a "historical ontology", considered here as the core of an original method that has proved to be a fundamental hermeneutic tool to the different ways of addressing socio-cultural contemporary issues. It also presents an interpretation of some of the key-concepts of Foucault's method formulations, especially "genealogy", "event", "modes of subjectivation", "forms of experience", "device" and "problematization". In addition to Foucault's own texts, which constitute the primary source of the paper, readings of interpreters of Foucault, who have made particularly indicative interventions on these concepts and problems, as Gilles Deleuze, Giorgio Agamben and Mathieu Potte-Bonneville, are used.
\end{abstract}

Key-words: Michel Foucault, historical ontology, socio-cultural analysis, genealogy, subjectivation.

\section{A "ontologia histórica" de Michel Foucault: anotações sobre o método para a análise crítica sociocultural}

\section{Resumo}

Este trabalho propõe uma reconstrução da perspectiva epistemológica e metodológica de Michel Foucault, ordenada em função do que ele caracterizou, no final de sua vida, como una "ontologia histórica”, considerada aqui como o núcleo específico de um método original que se revelou como uma ferramenta hermenêutica fundamental para as diferentes abordagens das problemáticas socioculturais contemporâneas. Para isso, apresenta-se uma interpretação de alguns dos conceitos-chave das formulações de método explicitadas por Foucault, especialmente a "genealogia", o "acontecimento" e os "modos de subjetivação", assim como as "formas de experiência", o "dispositivo" e a "problematização". Além dos textos do próprio Foucault, que constituem a fonte primordial do trabalho, se recorre às propostas de leitura de alguns intérpretes que têm realizado intervenções particularmente significativas a respeito desses conceitos e problemas, como Gilles Deleuze, Giorgio Agamben e Mathieu Potte-Bonneville.

Palavras-chave: Michel Foucault, ontologia histórica, análise sociocultural, genealogia, subjetivação.

Data de recebimento do artigo: 09/07/12

Data de aprovação do artigo: 31/10/12 